\title{
New results on the search for spin-exotic mesons with COMPASS
}

\author{
Frank Nerling ${ }^{* \dagger}$ \\ Physikalisches Institut, Albert-Ludwigs-Universität Freiburg, 79104 Freiburg, Germany \\ E-mail: nerling@eern.ch
}

The COMPASS fixed-target experiment at the CERN-SPS studies the structure and spectrum of hadrons. One important goal using hadron beams is the search for new states, in particular spinexotic mesons and glueballs. As a first input to the puzzle, COMPASS observed a significant $J^{P C}$ spin-exotic signal in the 2004 pilot run data $\left(190 \mathrm{GeV} / c \pi^{-}\right.$beam, $\mathrm{Pb}$ target $)$ in three charged pion final states consistent with the disputed $\pi_{1}(1600)$. We started our hadron spectroscopy programme in 2008 by collecting very high statistics using a $190 \mathrm{GeV} / \mathrm{c}$ negative pion beam scattered off a liquid hydrogen (proton) target. The current status and new results from the 2008 data on the search for the $\pi_{1}(1600)$ resonance with exotic $J^{P C}=1^{-+}$quantum numbers obtained from partial-wave analyses of the $\rho \pi$ and $\eta^{\prime} \pi$ decay channels are presented.

The 2011 Europhysics Conference on High Energy Physics, EPS-HEP 2011,

July 21-27, 2011

Grenoble, Rhône-Alpes, France

\footnotetext{
* Speaker.

$\dagger$ on behalf of the COMPASS collaboration
} 

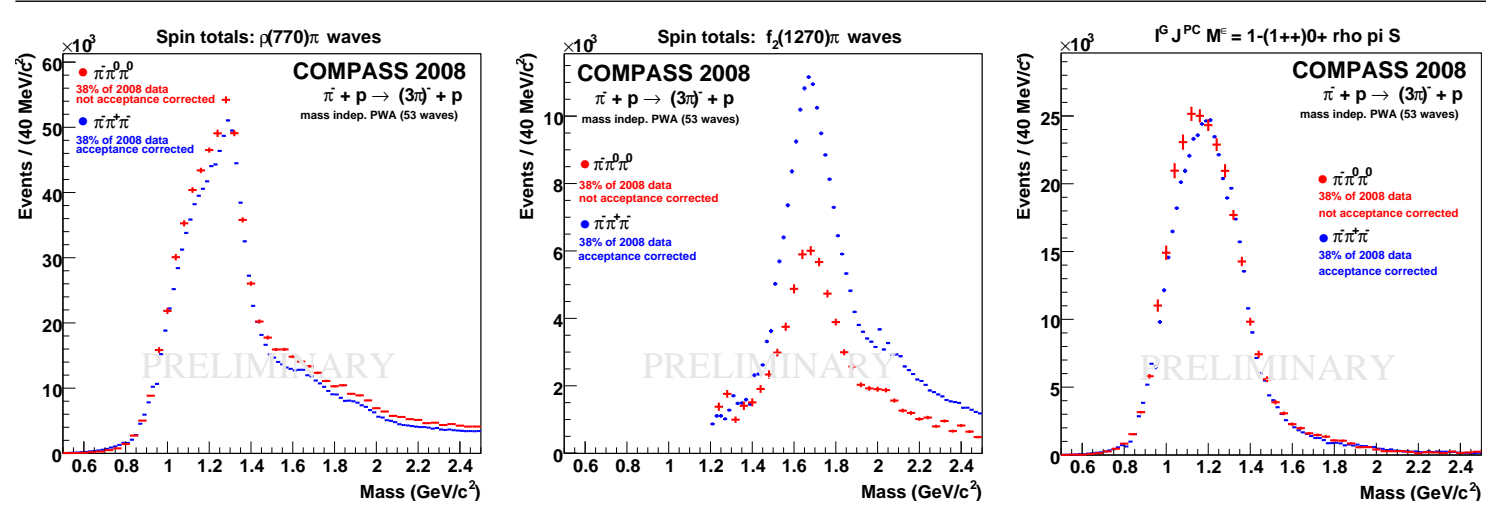

Figure 1: Intensity sums of all partial waves decaying into $\rho \pi$ (left) and $f_{2} \pi$ (centre) intermediate states included in the fits are in good agreement with expectations from isospin symmetry. The $a_{2}(1320)$ has been used for normalisation, see Fig. 2 (top, left). The data follows isospin symmetry not only for main waves, exemplary shown is the $a_{1}(1260)$ (right), but also for small ones, cf. Fig. 2, for discussion see text.

\section{Introduction}

The goal of the COMPASS experiment [1] at the CERN-SPS is to obtain a better understanding of the structure and dynamics of hadrons, both aspects of non-perturbative Quantum Chromodynamics (QCD). Given the accurate charged particle tracking as well as good coverage by electromagnetic calorimetry, the COMPASS 2008 hadron data provide an excellent opportunity for simultaneous observation of new states in different decay modes within the same experiment.

The existence of exotic states beyond the simple Constituent Quark Model (CQM), like socalled hybrids or glueballs, has been speculated about almost since the introduction of colour [2, 3]. Hybrid mesons are $q \bar{q}$ states with excited gluonic degree of freedom, whereas glueballs are purely gluonic states without valence quarks. They are both allowed within QCD (due to the selfcoupling of gluons via the colour-charge), while they are forbidden within the simple CQM. Glueball candidates have been reported by the Crystal Barrel and the WA102 experiments, however, the mixing with ordinary isoscalar mesons complicates the interpretation. Several light hybrids on the other hand are predicted to have exotic $J^{P C}$ quantum numbers and are thus promising candidates in the search for resonances beyond the CQM. The hybrid candidate lowest in mass is predicted [4] to have a mass between 1.3 and $2.2 \mathrm{GeV} / c^{2}$ and spin-exotic quantum numbers $J^{P C}=1^{-+}$, not attainable by ordinary $q \bar{q}$ states. In the light-quark sector, two $1^{-+}$hybrid candidates have been experimentally observed in the past in different decay channels, the $\pi_{1}(1400)$ mainly seen in $\eta \pi$ decays, by e.g. E852 [5], VES[6], and Crystal Barrel [7], and the $\pi_{1}(1600)$, observed by both E852 and VES in the decay channels: $\rho \pi[8,9], \eta^{\prime} \pi[6,10], f_{1} \pi[11,12,13]$, and $\omega \pi \pi[13,14]$. In $f_{1} \pi$, also a $\pi_{1}(2000)$ has been reported [12], however, this state still lacks any confirmation. In particular the resonant nature of the $\rho \pi$ decay channel of the $\pi_{1}(1600)$ observed in $3 \pi$ final states is highly disputed $[13,15]$. COMPASS has started to shed new light on the puzzle of spin-exotics by the observation of an $1^{-+}$signal in the 2004 data, consistent with the famous $\pi_{1}(1600)$. It shows clean phase motions with respect to other waves, confirming the resonance nature [16].

\section{New partial-wave analysis results on the search for the $\pi_{1}(1600)$}

COMPASS has access to all decay channels light spin-exotic mesons have been reported in so far, for a recent review see [17]. First PWA analyses focus on the $(3 \pi)^{-}$and $\eta^{\prime} \pi^{-}$final states, studying the existence of the contribution of the spin-exotic $1^{-+}$wave in the 2008 data. 

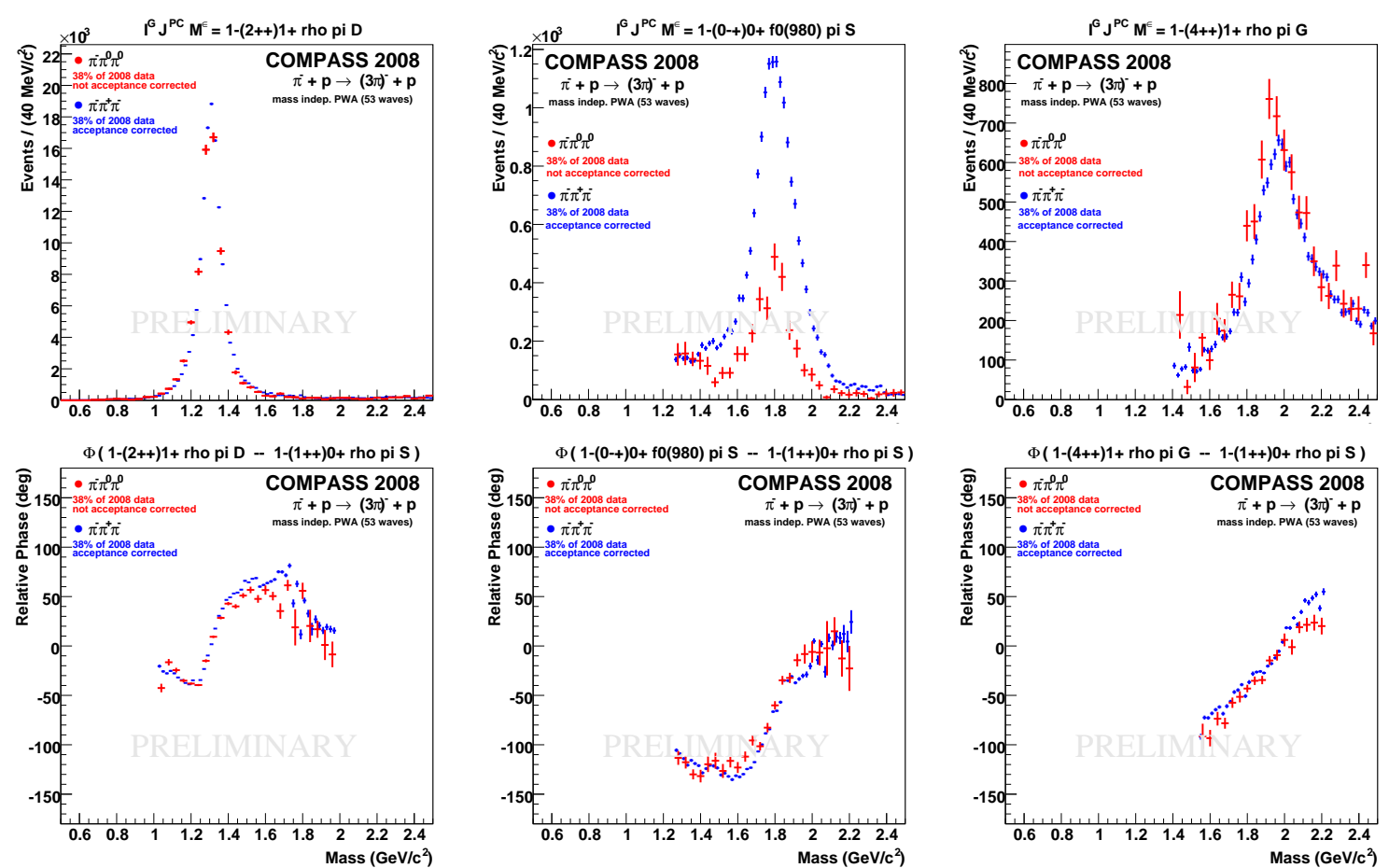

Figure 2: Intensities and relative phase differences $\Phi$ for main and small waves with respect to the prominent $a_{1}(1260)$. Exemplary shown are, from left to right, the $a_{2}(1320) \rightarrow \rho \pi$, the $\pi(1800) \rightarrow f_{0}(980) \pi$, and the $a_{4}(2040) \rightarrow \rho \pi$, respectively. The well-known $a_{2}(1320)$ resonance as well as the smaller, less prominent ones show a clean, rapid phase motion relatively to the $a_{1}(1260)$. Not only the intensities but also the phases are consistent for both, neutral $\left(\pi^{-} \pi^{0} \pi^{0}\right)$ and charged $\left(\pi^{-} \pi^{+} \pi^{-}\right)$decay modes, for discussion see text.

Diffraction of $\pi^{-}$into $(3 \pi)^{-}$final states - neutral and charged mode ( $\rho \pi$ decay channel) The new mass-independent PWA results for neutral and charged mode data presented in this paper, are normalised using the $a_{2}(1320)$ as a standard candle as shown in Fig. 2 (top, left) in order to compensate for the different detection efficiencies. This makes the fitted intensities for individual partial waves comparable between neutral and charged decay modes. A detailed description of the applied PWA method can be found in $[18,19]$ and references therein.

Due to the Clebsch-Gordan coefficients determining the different isospin coupling for the different underlying isobar structure, i.e. decays into an isovector versus an isoscalar, we expect for isospin 1 resonances decaying to $\rho \pi$ similar intensities for the neutral and charged mode data, whereas decays into $f_{2} \pi$ should show a suppression factor of two. Even though the neutral mode data have not yet been corrected for acceptance (similarly flat for the charged mode as already for the 2004 data), the data is in good agreement with these expectations from simple isospin coupling considerations. This holds to a large extent throughout the whole wave-set as shown by Fig. 1, depicting the intensity sums of all $\rho \pi$ (left) and $f_{2} \pi$ partial waves (centre), respectively. A few examples for fitted intensities of individual main and small waves are given in Fig. 1 (right), showing the $a_{1}(1260)$ decaying into $\rho \pi$ observed with same width and intensity for both decay modes, and Fig. 2, respectively, where also the relative phases with respect to the $a_{1}(1260)$ are shown. For the $\pi(1800)$ decay into $f_{0}(980) \pi$, we rather find about $45 \%$ instead of $50 \%$ for the intensity of the neutral as compared to the charged mode data, which is indeed expected taking into account effects from Bose-Symmetrisation, for a detailed discussion see [20]. 

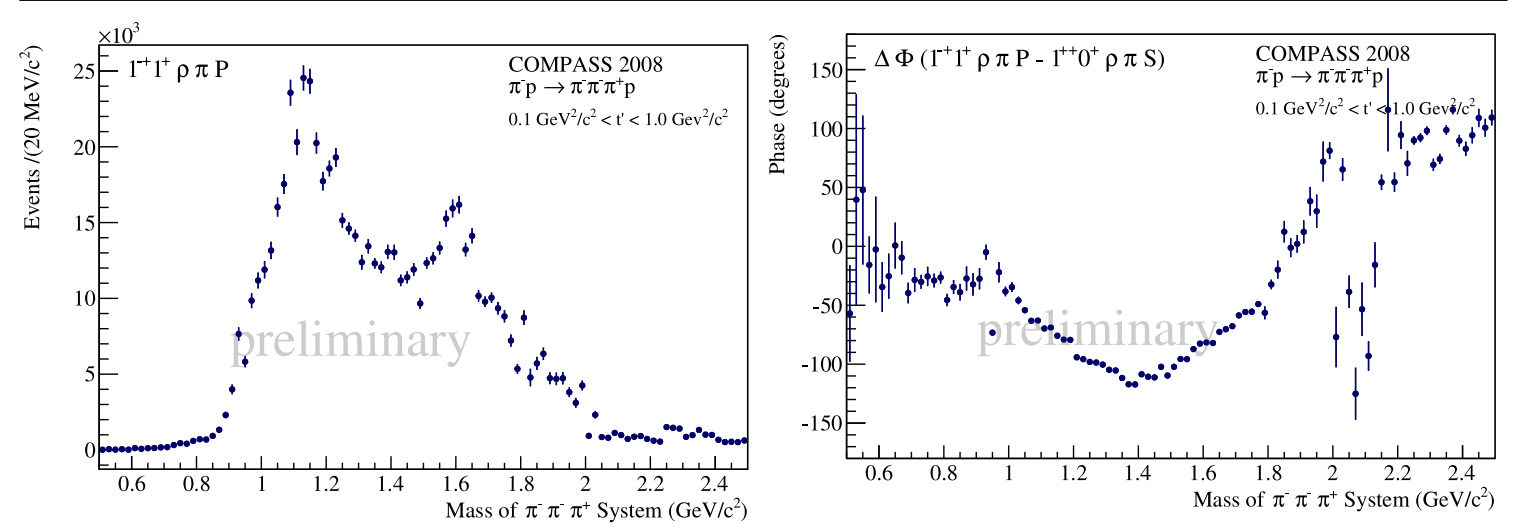

Figure 3: Intensity of the spin-exotic $1^{-+}$wave (left) and the phase difference with respect to the $a_{1}(1260)$.

The fitted intensities of the spin-exotic $1^{-+}$wave and the relative phase again with respect to the $a_{1}(1260)$ resonance are shown for the charged mode (Fig. 3). This wave shows a structure at about $1.6 \mathrm{GeV} / c^{2}$. A raising phase is observed in the corresponding mass region, consistent with the structure in the exotic wave at $1.6 \mathrm{GeV} / c^{2}$ having resonant nature and resonating against the tail of the $a_{1}(1260)$. This is consistent with the result of the PWA of the 2004 pilot run data taken with a $\mathrm{Pb}$ target [16]. The bump at around $1.2 \mathrm{GeV} / \mathrm{c}^{2}$ needs further to be understood, it appears unstable with respect to changes in the PWA model, and is still under investigation [19].

Diffraction of $\pi^{-}$into $\pi^{-} \pi^{+} \pi^{-} \eta$ final states ( $\eta^{\prime} \pi$ decay channel) Reconstructing $\pi^{-} \pi^{+} \pi^{-} \eta$ final states, the diffractively produced $\pi^{-} \eta^{\prime}$ system can be analysed using PWA. The involved decay chain is here $\eta^{\prime} \rightarrow \pi^{+} \pi^{-} \eta, \eta \rightarrow \gamma \gamma$, and the resulting sub-mass spectrum, Fig. 4 (top, left),
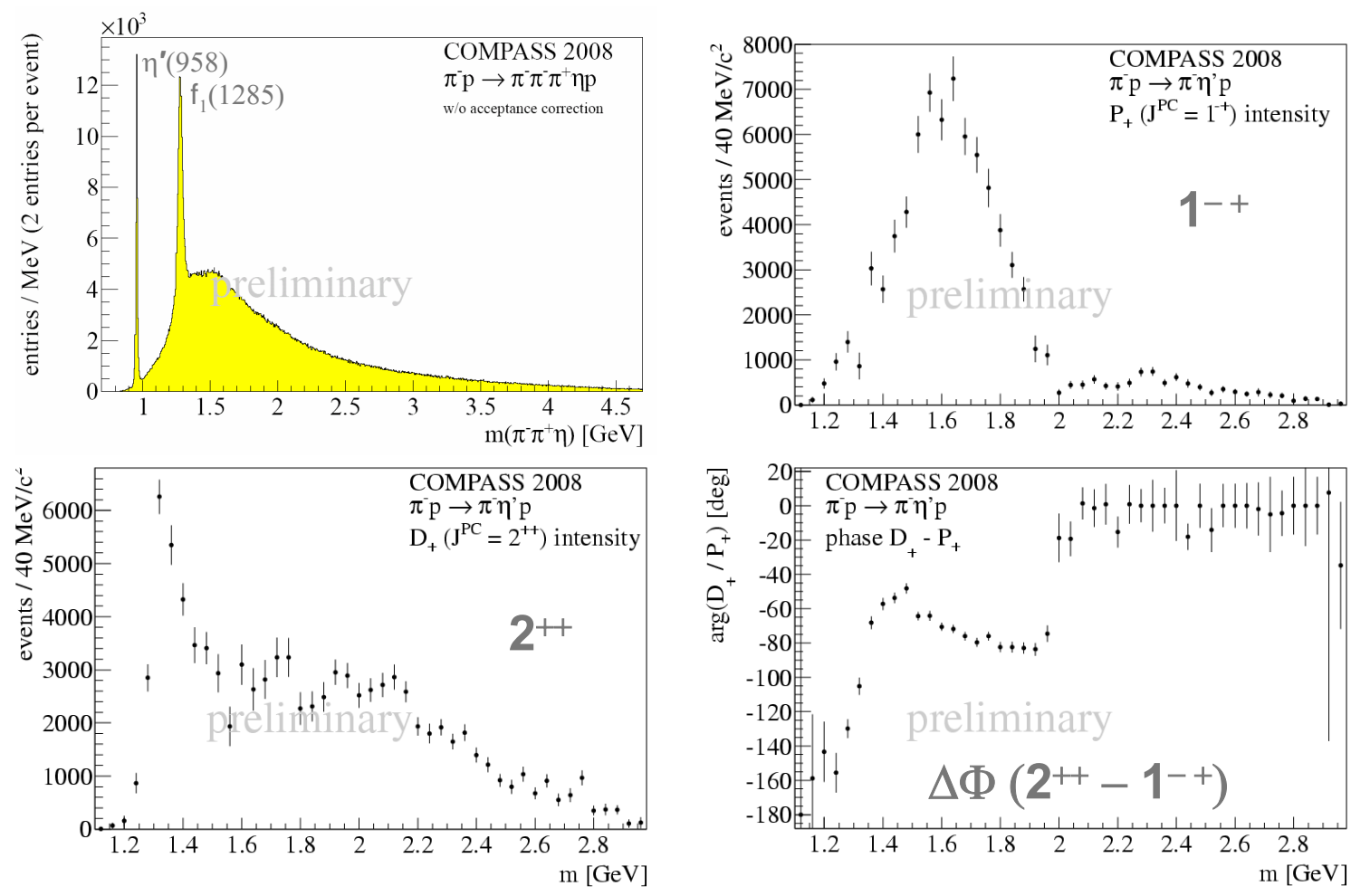

Figure 4: Top: Invariant sub-mass spectrum showing $\eta^{\prime}(958)$ and $f_{1}(1285)$ peaks (left), and the massindependently fitted intensities of the spin-exotic $1^{-+}\left(P_{+}\right)$wave (right). Bottom: Fitted intensities of the $2^{++}\left(D_{+}\right)$wave showing the $a_{2}(1320)$ (left) and the relative phase $D_{+}-P_{+}$(right). 

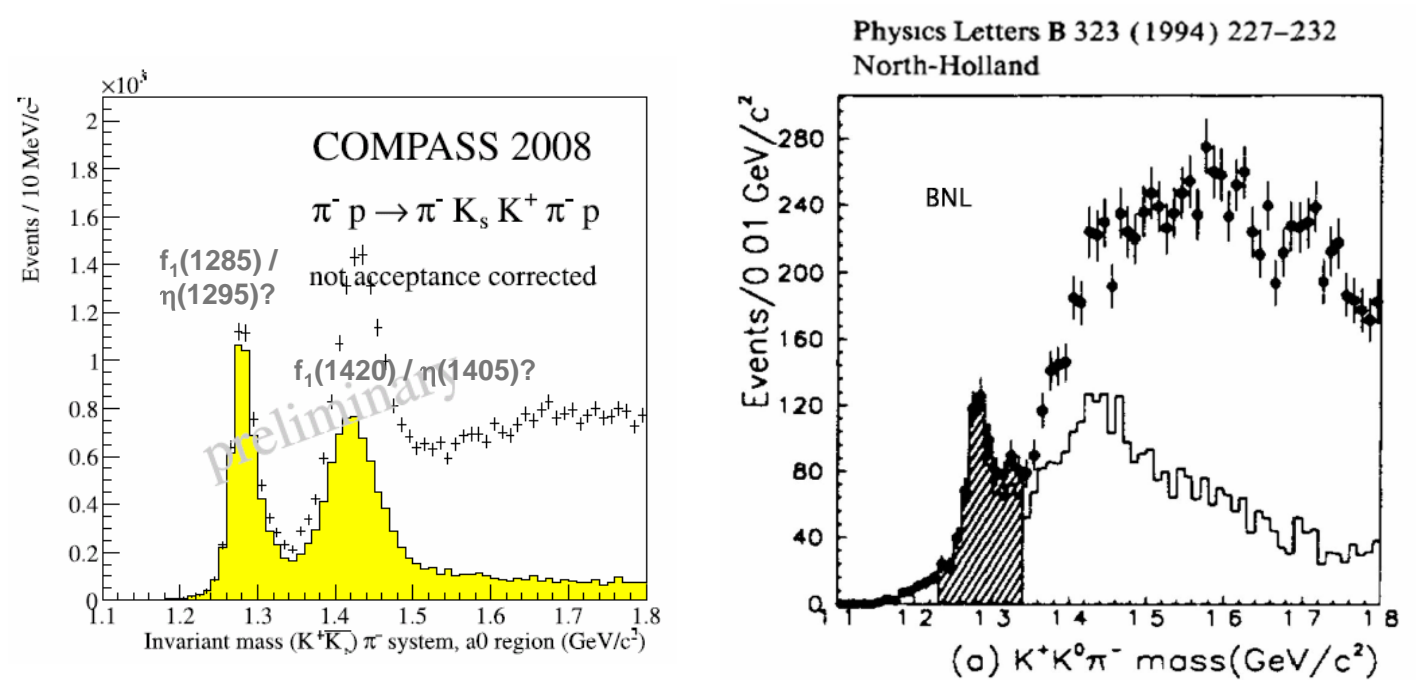

Figure 5: The $(K \bar{K} \pi)^{0}$ sub-mass system shows clean $f_{1}(1285)$ and $f_{1}(1420)$ peaks before (dots) and after (line) an additional restriction of the $(K \bar{K})$ mass to the $a_{0}(980)$ region. Comparing the similar plot obtained at BNL [11] (right), the COMPASS statistics exceeds the one analysed by E852 by a factor of 10, even a factor of 20, taking into account also the 2009 data taken with $\pi^{-}$beam. Not only the observed $f_{1}(1285)$ but also the $f_{1}(1420)$ are nearly background free as compared to the published result by BNL/E852 [11].

exhibits clean, narrow $\eta^{\prime}(958)$ and $f_{1}(1285)$ peaks. The mass-independent PWA follows previous analyses, $P_{+}$and $D_{+}$waves are included as well as a $G_{+}$wave in addition [21]. A clear signal of the $a_{2}(1320)\left(D_{+}\right)$appears, and the most intense wave is the spin-exotic $\left(P_{+}\right)$wave, showing a broad structure at around $1.6 \mathrm{GeV} / c^{2}$ consistent with other experiments. More systematic studies are, however, needed before strong conclusions can be drawn, the sharp jump in the phase difference $\left(D_{+}-P_{+}\right)$at about $2 \mathrm{GeV} / c^{2}$ has e.g. further to be understood.

Diffraction of $\pi^{-}$into $K_{s}^{0} K^{ \pm} \pi^{\mp} \pi^{-}$final states $\left(f_{1} \pi\right.$ decay channel) - Outlook The $(K \bar{K} \pi)^{0}$ subsystem shown in Fig. 5 (exemplary for one case) is of particular interest, as spin-exotic $1^{-+}$ resonances were reported in the $f_{1} \pi$ decay channel. The COMPASS data feature clean $f_{1}(1285)$ and $f_{1}(1420)$ peaks (Fig. 5). Even though an $\eta$ contribution cannot be excluded, a first massindependent PWA indicate contributions from $\eta(1405)$ and $\eta(1295)$ to be minor, consistent with the observation by E852 [12], for further details, see [22]. The complementary possibility for studying the $f_{1} \pi$ decay channel are $\eta 3 \pi$ final states, also analysed by E852. This channel is feasible (Fig. 4, top/left) and will be analysed by COMPASS as well.

\section{Conclusions \& summary}

The high statistics hadron data will allow COMPASS to contribute solving the puzzle of light spin-exotic mesons. The new results presented on the $\rho \pi$ decay channel in both, the neutral and charged decay modes of the $(3 \pi)^{-}$system, appear very consistent and solid not only for main but also for small waves. There is presently no contradiction between both analyses results. The result from the 2004 pilot run data seems on the way to be confirmed, more systematic studies are needed before doing the mass-dependent PWA analyses. In-line with other experiments, a huge contribution of the spin-exotic $1^{-+}$wave in the $\eta^{\prime} \pi$ decay channel is confirmed in the COMPASS data, even though more systematic studies are needed before conclusions on the resonant nature can be drawn. The feasibility for spin-exotic search in the $f_{1} \pi$ decay channel in diffractively produced $K \bar{K} \pi \pi$ final states has been shown, not only for $f_{1}(1285) \pi$ but also for a first study of the 
$f_{1}(1420) \pi$ system, never done before. Apart of the results discussed in this paper, COMPASS has all important decay channels for spin-exotic search at sufficient statistics on tape, and the recent results presented here confirm the excellent potential to conclude on the existence of the highly disputed spin-exotic $\pi_{1}(1600)$ resonance and others.

Acknowledgements This work is supported by the BMBF (Germany), in particular via the "Nutzungsinitiative CERN".

\section{References}

[1] P. Abbon et al., COMPASS collaboration, Nucl. Instr. Meth. A 68 (2007) 455.

[2] R. Jaffe and K. Johnsons, Phys. Lett. B 60 (1976) 201.

[3] T. Barnes et al., Nucl. Phys. B 224 (1983) 241.

[4] K.J. Juge, J. Kuti, C. Morningstar, AIP Conf. Proc. 688 (2004) 193.

[5] D. R. Thomson et al., Phys. Rev. Lett. 79 (1997) 1630.

[6] G. M. Beladidze et al., Phys. Lett. B 313 (1993) 276.

[7] A. Abele et al., Phys. Lett. B 423 (1998) 175.

[8] G. S. Adams et al., Phys. Rev. Lett. 81, (1998) 5760.

[9] Y. Khokhlov, Nucl. Phys. A 663 (2000) 596.

[10] E. I. Ivanov et al., Phys. Rev Lett. 86 (2001) 3977.

[11] J.H. Lee et al., Phys. Lett. B 323 (1994) 227.

[12] J. Kuhn et al., Phys. Lett. B 595 (2004) 109.

[13] D. V. Amelin et al., Phys. Atom. Nucl. 68 (2005) 359.

[14] M. Lu et al., Phys. Rev. Lett. 94 (2005) 032002.

[15] A.R. Dzierba et al., Phys. Rev. D 73 (2006) 072001.

[16] M. Alekseev et al., COMPASS collaboration, Phys. Rev. Lett, 104 (2010) 241803.

[17] C.A. Meyer and Y.Van Haarlem, Phys. Rev. C 82 (2010) 025208; arXiv:1004.5516v2 [nucl-ex].

[18] F. Nerling, AIP Conf. Proc. 1257 (2010) 286; arXiv:1007.2951 [hep-ex].

[19] F. Haas, Conf. Proc. Hadron2011, Munich, Germany (2011).

[20] F. Nerling, Conf. Proc. Hadron2011, Munich, Germany (2011); arXiv:1108.5969 [hep-ex].

[21] T. Schlüter, Conf. Proc. Hadron2011, Munich, Germany (2011); arXiv:1108.6191 [hep-ex].

[22] J. Bernhard and F. Nerling, Conf. Proc. Hadron2011, Munich, Germany (2011); arXiv:1109.0219 [hep-ex]. 